\title{
CORRECTION
}

View Article Online

View Journal I View Issue

\section{Correction: The effect of recombination under short-circuit conditions on the determination of charge transport properties in nanostructured photoelectrodes}

Cite this: Phys. Chem. Chem. Phys., 2016, 18, 14139

\author{
J. Villanueva-Cab, ${ }^{\star a}$ J. A. Anta ${ }^{b}$ and G. Oskam ${ }^{c}$
}

DOI: $10.1039 / c 6 c p 90121 b$

www.rsc.org/pccp

Correction for 'The effect of recombination under short-circuit conditions on the determination of charge transport properties in nanostructured photoelectrodes' by J. Villanueva-Cab et al., Phys. Chem. Chem. Phys., 2016, 18, 2303-2308.

On page 2304 of the published article, eqn (1) should be amended as shown below:

$$
\frac{\partial n}{\partial t}=\frac{\partial}{\partial x}\left[D_{0}\left(n / n_{0}\right)^{(1-\alpha) / \alpha} \frac{\partial n}{\partial x}\right]+G(x)-k_{0}\left(n / n_{0}\right)^{(\beta-\alpha) / \alpha} n
$$

In addition, Fig. 2 in the published article should be replaced with the revised version below:

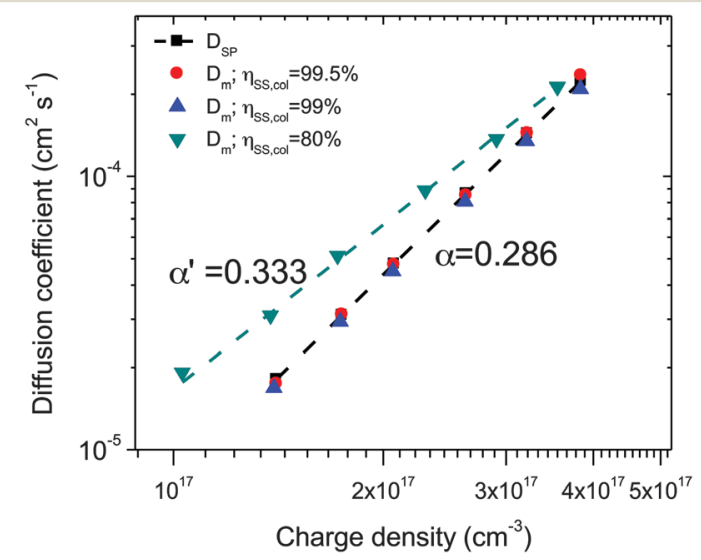

Fig. 2 Diffusion coefficient as function of charge density. Comparison between measured (SLIT) values and the model predictions for three different recombination rate constants: $k_{0}=0$ ( $\eta_{\mathrm{ss}, \text { coll }}=99.5 \%$ at 1 Sun $), k_{0}=2.718 \times 10^{-3} \mathrm{~s}^{-1}\left(\eta_{\mathrm{ss}, \text { coll }}=99 \%\right.$ at 1 Sun $)$ and $k_{0}=0.12 \mathrm{~s}^{-1}\left(\eta_{\mathrm{sS}, \mathrm{coll}}=80 \%\right.$ at $\left.1 \mathrm{Sun}\right)$. When recombination takes place under short circuit conditions, the diffusion coefficient is overestimated, which can be erroneously interpreted as a trap distribution of $\alpha^{\prime}=0.333$. See the text for details.

The Royal Society of Chemistry apologises for these errors and any consequent inconvenience to authors and readers.

\footnotetext{
${ }^{a}$ Instituto de Física, Benemérita Universidad Autónoma de Puebla, Apdo. Postal J-48, Puebla, Pue. 72570, Mexico. E-mail: juliovc@ifuap.buap.mx

${ }^{b}$ Nanostructured Solar Cells Group, Department of Physical, Chemical and Natural Systems, Universidad Pablo de Olavide, Ctra. Utrera, km 1, ES-41013 Seville, Spain

${ }^{c}$ Departamento de Física Aplicada, CINVESTAV-IPN, Mérida, Yuc. 97310, Mexico
} 